\title{
Evaluation of a policy of lymph node retrieval for colon cancer specimens: a quality improvement opportunity
}

\author{
Stephen Jones, MD, ${ }^{a}$ Robert Mazziotta, MD, ${ }^{\mathrm{b}}$ and Lawrence E Harrison, MD ${ }^{\mathrm{a}}$
}

Departments of a Surgery and ${ }^{b}$ Pathology, The Daniel and Gloria Blumenthal Cancer Center, Valley Health System, One Valley Health Plaza, Paramus, New Jersey

Background In an effort to improve compliance with the national guidelines of adequate lymph node harvest for colon cancer, the Department of Pathology at the Valley Health System in Paramus, New Jersey, established a policy in 2011 stating that if fewer than 12 lymph nodes were evaluated after initial dissection of a non-metastatic invasive colon cancer specimen, then re-dissection of the specimen was performed to harvest additional lymph nodes.

Objective To evaluate the efficacy of the policy as it relates to the compliance for sufficient lymph node evaluation in non-metastatic invasive colon cancers.

Methods A review of the Valley Hospital Health System Tumor Registry for all adult patients who had undergone surgery for stages I-III colon adenocarcinoma during January 1, 2007-July 1, 2015 identified 626 patients. The patients were divided into 2 groups, pre-policy $(n=301)$ and post-policy ( $n=325)$, for analysis.

Results The median lymph node yield in the post-policy group increased significantly and the percentage of inadequate lymph node evaluation significantly decreased, compared with the pre-policy group. With the improvement of lymph node yields, fewer patients received chemotherapy for stage II colon cancer. Overall survival of patients with adequate lymph node yields was significantly longer than in patients with inadequate yields.

Limitations Single institutional study with relatively small numbers.

Conclusions The results of this study suggest that the implementation of a policy of reflexive re-dissection for colon cancer specimens with inadequate lymph node yields decreases the number of insufficient lymph node specimens and significantly improves compliance with national guidelines.

$\mathrm{C}$ olorectal cancer is the third leading cause of cancer deaths in the United States. ${ }^{1}$ Lymph node status remains a significant predictor of survival and is of crucial importance for both prognosis and stratification for adjuvant treatment. National guidelines recommend that at least 12 lymph nodes be resected and pathologically evaluated for patients with non-metastatic colon cancer. $^{2-6}$ The implications of adequate lymph node yield can be seen in numerous studies, which demonstrate a correlation with increasing number of lymph nodes examined with overall and diseasespecific survival. ${ }^{7-10}$ Although the direct therapeutic benefit from lymph node dissection is not proven, the documented improvement in survival associated with adequate lymph node retrieval is most likely related to stage migration and appropriate use of effective adjuvant therapy.

The threshold of evaluating 12 lymph nodes is an accepted quality measure for the treatment of patients with colon cancer, reflecting on both the adequacy of surgical resection, as well as the completeness of pathologic evaluation. Although the recommendation that at least 12 lymph nodes be resected and pathologically evaluated for patients with colon cancer has been disseminated for over a decade, compliance remains suboptimal at many institutions. ${ }^{11-13}$ Multiple factors have been cited for non-compliance, including those relating to the patient, surgeon, and pathologist. ${ }^{14}$

To improve compliance with the national guidelines of adequate lymph node harvest, the Department of Pathology at the Valley Health System in Paramus, New Jersey, established a policy in 2011 stating that if fewer than 12 lymph nodes were evaluated after initial dissection of a non-metastatic invasive colon cancer specimen, a re-dissection of the specimen should be reflexively performed to harvest any additional lymph nodes missed at the time of first evaluation. The objective of this study is

Accepted for publication June 30, 2016. Correspondence: Lawrence E Harrison, MD; Lawrence.Harrison@Atlantichealth. org. Disclosures: The authors report no disclosures or conflicts of interest. JCSO 2016;14(9):380-385. (02016 Frontline Medical Communications. doi: 10.12788/jcso.0285. 
to evaluate the efficacy of that policy as it relates to lymph node harvest and pathologic evaluation and to assess whether it had an impact on the compliance for sufficient $(\geq 12)$ lymph node evaluation in non-metastatic invasive colon cancers.

\section{Methods}

A review of the Valley Hospital Health System Tumor Registry for all adult patients who had undergone surgery for colon adenocarcinoma from January 1, 2007 to July 1, 2015 identified 805 patients. Patients with rectal cancer were excluded from this study. Of the 805 patients, 39 underwent resection for Tis disease (stage 0), an additional 130 patients underwent surgery for synchronous metastatic disease (stage IV), and 10 patients had unknown staging and were excluded from the analysis. The final study population consisted of 626 patients, which included all patients with resected stage I, II, or III colon adenocarcinomas over the 8-year period.

Before 2011, there were no established standards at our institution regarding lymph node retrieval for resected colon adenocarcinoma specimens. In 2011, a policy was initiated within the Department of Pathology whereby, if there were fewer than 12 lymph nodes evaluated after initial review of a colon cancer specimen, a re-dissection of the specimen was reflexively performed to harvest any additional lymph nodes missed at the time of first evaluation.

\section{Statistical analysis}

Statistical analysis was performed using SPSS 13.0 (SPSS Inc, Chicago, IL). Data are expressed as median values (range). Survival was calculated by the method of Kaplan-Meier and compared by log rank test. Comparisons were by unpaired $t$ or chisquare test where appropriate, with statistical significance defined as $P<.05$.

\section{Results}

Patients with stage I, II or III colon cancers ( $\mathrm{n}=$ 626) accounted for $77.8 \%$ of the 805 colon cancer cases treated at the hospital during the 8-year period. The patients were divided into 2 time periods, based on the initiation of the lymph node retrieval policy. During January 1, 2007-December 31, 2010, 301 patients underwent resection for stage I-III colon cancers, and 325 underwent resection for the same stages of disease from January 1, 2011 to July 15, 2015 (Table 1). The median number of lymph nodes retrieved in the pre-policy group was 15 (range, 0-66), compared with 18 (range, 0-53) in the post-policy group $(P=.04)$. In addition, the number of cases with inadequate number of evaluated lymph nodes ( $<12$ nodes) decreased significantly, from $27.2 \%$ in the pre- policy group to $10.4 \%$ in the post-policy group $(P=.001$, Table 1). Despite improvements in median nodal yield and a decrease in inadequate lymph node yields, stage distribution did not change when comparing the 2 time periods. However, we found an increase in the number of node-positive patients for the group that had fewer than 12 lymph nodes evaluated when the entire cohort of 626 patients was included (Table 2). In patients with stage II disease, a significantly greater percentage $(41.2 \%)$ received chemotherapy when fewer than 12 lymph nodes were evaluated, compared with patients with adequate staging (17.1\%; Table 3).

The number of lymph nodes retrieved per specimen
TABLE 1 Review of tumor registry, stages H-III colon cancer (2007-2015a) See note below

\begin{tabular}{llll} 
& \multicolumn{2}{c}{ Group } & \\
\cline { 2 - 3 } Characteristic & Pre-policy, & Post-policy, & \\
& $2007-2010^{b}$ & $2011-2015 c$ & P value \\
& $(n=301)$ & $(n=325)$ &
\end{tabular}

$\begin{array}{cccc}\text { Stage } & & & \\ 1 & 91 & 77 & \text { NS } \\ \text { II } & 104 & 134 & \text { NS } \\ \text { III } & 106 & 114 & \text { NS }\end{array}$

Total $\quad 301 \quad 325$

Median no. lymph nodes, $\mathrm{n}$ (range)

Proximal colon $\quad 17(0-51) \quad 19(0-48) \quad .04$

Distal colon $\quad 13(0-66) \quad 17(0-53) \quad .03$

Total $\quad 15(0-66) \quad 18(0-53) \quad .04$

Insufficient nodal yield (<12), n (\%)

$\begin{array}{llll}\text { Proximal colon } & 23(16.6) & 11(7.7) & .02 \\ \text { Distal colon } & 59(36.4) & 23(12.7) & .001 \\ \text { Total } & 82(27.2) & 34(10.4) & .001\end{array}$

NS, not significant

aJanuary 1, 2007 to July 15, 2015. bJanuary 1, 2007-December 31, 2010. January 1, 2011 1-July 15, 2015.
TABLE 2 Lymph node retrieval and impact on node positivity

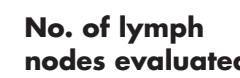

\begin{tabular}{cc}
\multicolumn{3}{c}{ Stage, $\mathbf{n}(\%)^{\mathbf{a}}$} \\
\hline I and II & III \\
88 & $28(25.0)$ \\
318 & $192(37.6)^{*}$ \\
406 & 220
\end{tabular}

Percentages are rounded.

$\star P=.007$ 


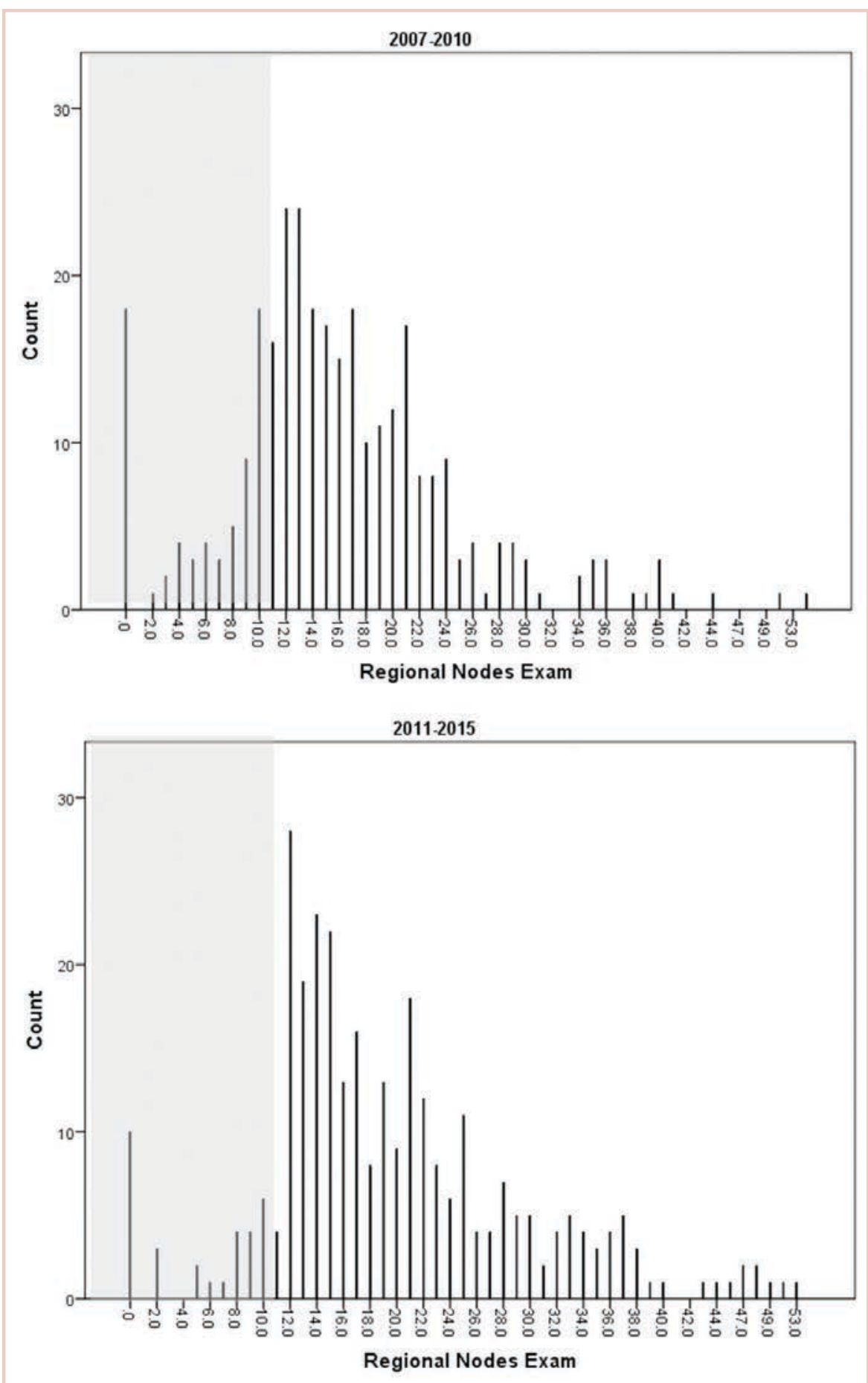

FIGURE 1 Distribution of lymph nodes per specimen. The histograms compare the number of lymph nodes retrieved per specimen for the pre- and post-policy periods. The gray shaded areas highlight patients with inadequate lymph node retrieval. A comparison of the gray areas in panel A (pre-policy) and panel B (post-policy) shows that the number of patients with inadequate lymph node retrieval decreased in the post-policy period.

increased from the pre- to post-policy period (Figure 1), and the number of patients with inadequate retrieval significantly decreased from the pre- to post-policy period
(Figure 1). Although there was no difference in survival between the pre- and -post policy periods (data not shown), a significant increase in overall survival was noted with patients who had $\geq 12$ lymph nodes evaluated, compared with those who had $<12$ lymph nodes evaluated (Figure 2). When the percentage of the patients with inadequate lymph nodes retrieved were evaluated by study year, there was a steady decrease from 36.3\% in 2007 (pre-policy) to $11.4 \%$ by 2011 , which remained relatively stable to 2015, the end of the post-policy period (Figure 3).

Tumor location also had an impact on lymph node retrieval. Overall, proximal colon (ascending and transverse) specimens had a median of 18 lymph nodes (range, 0-51), which was significantly greater than distal colon (splenic flexure to rectosigmoid) specimens (15 lymph nodes [range, $0-66] ; P=.05)$. When the number of lymph nodes was evaluated based on time period, the median number of lymph nodes was higher in proximal lesions compared with distal lesions. Similarly, the number of patients with insufficient nodal yields was higher in distal tumors (Table 1). Although both sites improved over time, the improvement in the number of patients with inadequate nodal staging was more dramatic for the distal tumors, compared with the proximal lesions (Figure 4).

The entire cohort of 626 patients was evaluated based on the number of lymph nodes retrieved and correlated with lymph node status (stage I and II [node negative] compared with stage III [node positive]). When 12 or more lymph nodes were pathologically evaluated, 37.6\% (192 of 510) of the patients had positive lymph nodes (stage III), whereas when fewer than 12 nodes were evaluated, $24.1 \%$ (28 of 116) had stage III tumors $(P=.007$; Table 2$)$. One relative indication for adjuvant systemic chemotherapy for node negative colon cancers is inadequate lymph node retrieval. Of the 238 patients with stage II disease who were evaluated, chemotherapy data was available for 175 patients. When fewer than 12 lymph nodes were evaluated in this subset of stage II colon cancer patients, 41.2\% (7 of 17 patients) received systemic chemotherapy. The number of patients receiving chemotherapy decreased to $17.1 \%(27$ 


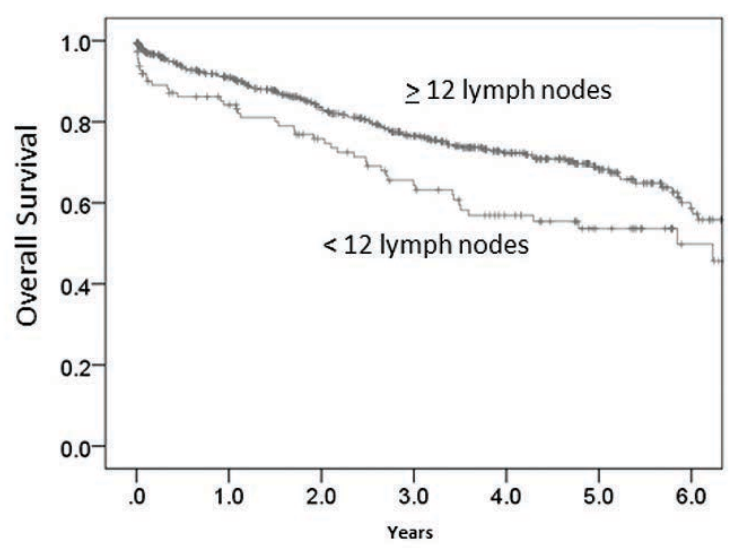

FIGURE 2 Overall survival of patients based on adequate lymph node evaluation. A significant increase in overall survival was noted in patients who had $\geq 12$ lymph nodes evaluated, compared with patients who had $<12$ lymph nodes evaluated $(P=.05)$.

of 158 patients) in node-negative patients with adequate lymph node evaluation $(P=.03$; Table 3$)$.

\section{Discussion}

Lymph node staging is a powerful predictor of survival and is important in selecting patients for adjuvant chemotherapy for patients with non-metastatic colon cancer. Based on the findings from multiple studies that have correlated lymph node retrieval with overall survival, ${ }^{8,10,15}$ numerous organizations, including the American College of Surgeons, American Society for Clinical Oncology, the National Comprehensive Cancer Network, and The National Quality Forum have endorsed the evaluation of a minimum of 12 lymph nodes as a hospital quality assessment standard. Despite these recommendations, compliance to this metric has varied considerably. Although there has been improvement over time at some hospitals, compliance is still inadequate. ${ }^{5,12}$

Multiple variables influence lymph node retrieval and have been well summarized in the literature. ${ }^{14}$ However, factors specific to the surgeon, pathologist, and hospital, rather than to patient or tumor characteristics, are important to identify, because they can be modified to implement quality improvement initiatives.

The current study evaluated a hospital-based department of pathology policy in order to improve compliance with the national guidelines of adequate lymph node harvest. Starting at a peak of $35 \%$ inadequate retrieval rate in 2007 , our hospital rate of insufficient node retrieval was about $25 \%$ in the pre-policy time period. With the implementation of the policy, the rate of insufficient lymph node retrieval decreased significantly to $10.4 \%$ (Table 1 ). Although the trend during the pre-policy period, from 2007-2010, shows a gradual decrease in inadequate lymph node yield, there

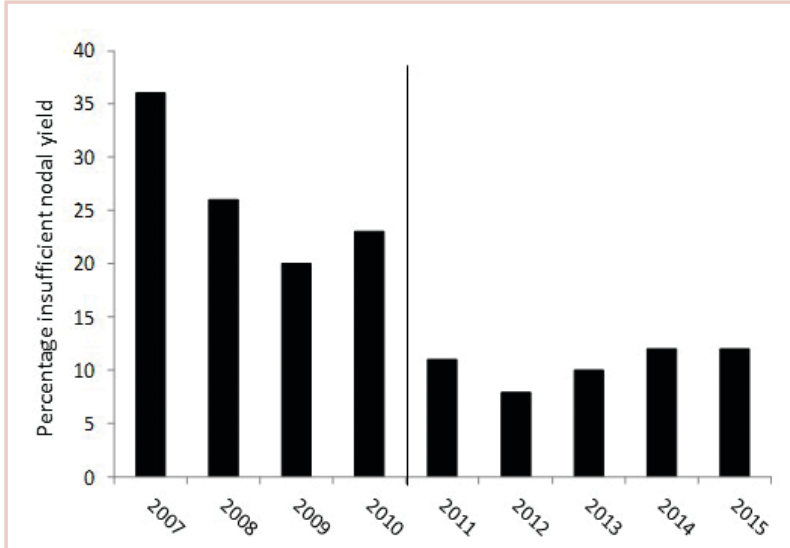

FIGURE 3 Percentage of inadequate lymph node evaluation cases by year. The percentage of cases with inadequate lymph nodes decreased from nearly $35.3 \%$ in 2007 to $7.4 \%$ in 2011 , and remained stable through 2015.

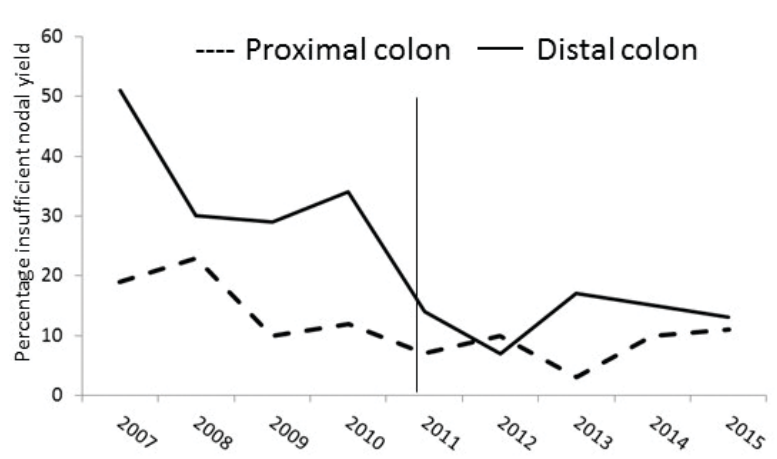

FIGURE 4 Percentage of inadequate lymph node evaluation cases by tumor site. Although proximal and distal lesions improved over time, the decrease in the number of patients with inadequate nodal staging was more dramatic for the distal tumors, compared with the proximal lesions.

TABLE 3 Stage II colon cancer and adjuvant chemotherapy

\begin{tabular}{lcc|}
$\begin{array}{c}\text { No. of lymph } \\
\text { nodes evaluated }\end{array}$ & $\begin{array}{c}\text { No chemotherapy, } \\
\mathbf{n}(\%)^{\mathbf{a}}\end{array}$ & $\begin{array}{c}\text { Chemotherapy, } \mathbf{n} \\
(\%)^{\mathbf{a}}\end{array}$ \\
$<12(\mathrm{n}=17)$ & 10 & $7(41.2)$ \\
$\geq 12(\mathrm{n}=158)$ & 131 & $27(17.1)^{*}$ \\
\hline Total $(\mathrm{N}=175)$ & 141 & 34 \\
\hline $\begin{array}{l}* P=.003 \\
\text { apentages are rounded. }\end{array}$ & & \\
\end{tabular}

was a more noticeable and persistent decrease after policy implementation (Figure 2). We speculate that the initial decrease observed during the pre-policy period was due to an overall heightened national emphasis on lymph node retrieval. However, after the policy was in place, the num- 
ber of insufficient nodal retrieval cases decreased significantly and remained stable over time. We did not document the number of times a specimen was re-evaluated after the policy was in place, however, the significant and sustained compliance that was observed in the post-policy period may have been the result of a combination of factors in addition to reflexive re-dissection, including an improved initial dissection by pathology technicians who understand the implications of an inadequate first lymph node dissection.

Although the policy of re-dissection for inadequate retrieval has resulted in an improvement in our hospital compliance, about $10 \%$ of specimens remain with inadequate lymph node assessment. It is possible that by optimizing the pathology side of the equation, the remaining percentage may, in part, be surgeon dependent. Nathan and colleagues used the SEER database from 1998-2005 to evaluate the lymph node assessment for patients undergoing curative colon resection. Based on their multivariable model analysis, they concluded that the surgical technique accounted for $8.2 \%$ of cases with inadequate lymph node retrieval, ${ }^{13}$ a number similar to the remaining cases observed in our series. Non-modifiable factors, such as patient age, distribution of tumor site, and the presence of microsatellite instability may also contribute to the remaining inadequate nodal yields. ${ }^{16,17}$

The impact on achieving adequate lymph node staging is exemplified by the significantly increased number of patients who received systemic chemotherapy associated with inadequate lymph node yields. Although there are no data to support routine use of adjuvant chemotherapy for node negative colon cancer, there are a subset of these patients who may at higher risk of recurrence and therefore, postoperative treatment. One such relative indication is inadequate lymph node staging. ${ }^{18} \mathrm{We}$ observed that for patients with stage II disease, a significantly greater percentage (41.2\%) received chemotherapy when fewer than 12 lymph nodes were evaluated, compared with patients with adequate staging (17.1\%). Although this is an important finding, there are limitations to the data, including an incomplete data set (63 patients with stage II tumors did not have information about chemotherapy), as well as not accounting for other high-risk factors in this set of patients, which may have contributed to whether they received chemotherapy.

Despite improvements in median nodal yield and a decrease in inadequate lymph node yields, stage distribution did not change when comparing the 2 time periods. Although it may seem counterintuitive, that finding has been reported by others. ${ }^{19,}{ }^{20} \mathrm{We}$ did, however, document an increase in the number of node-positive patients for the group that had fewer than 12 lymph nodes evaluated when the entire cohort of 626 patients was analyzed. This finding may represent stage migration as a result of improved nodal yield, but it is also possible that the significance noted may be due to the relatively low number of node-positive patients in the inadequate lymph node staging group (25\%). By comparison, other investigators have documented a stage III distribution of about 35\%-40\%, regardless of the number of lymph nodes retrieved. ${ }^{19,21}$

The Valley Hospital is a high-volume community hospital, performing more than 40 colon cancer resections annually, with most of the colon cancer surgery performed by specialty trained colorectal surgeons or surgical oncologists. All pathology specimens are dissected by full-time technologists. The strength of the current study is that these variables remained relatively constant throughout the study period. The only major change over the 8-year study period was the implementation of the specimen dissection policy in 2011. Therefore, the ability to assess the impact of that policy is not confounded by other concomitant variables. However, there are limitations to this study. Data collection for the pre- and post-policy periods was retrospective, which introduces obvious biases. In addition, during the 8-year period, there were changes in the management of patients with colon cancer, which may have had an impact on overall survival, independent of lymph node retrieval. In addition, the number of specimens requiring re-dissection was not documented. The number of re-dissections would have been an important data point to have for this study to further demonstrate the impact of the policy. It is our impression that with a heightened sense of expectations to achieve adequate lymph node retrival after the impletnation of this policy, the need for re-dissection has decreased over time, but we do not have data to support this conclusion.

Although the assessment of 12 lymph nodes as metric adequate staging is embraced by many national organizations, others have questioned its utility as a quality-of-care measure. ${ }^{22}$ Stage distribution does not seem to be affected by improving nodal yields and factors, such as age and tumor site, and tumor factors such as microsatellite instability and tumor infiltrating lymphocytes are not modifiable. However, until a better metric is identified, it will be important to evaluate 12 or more lymph nodes in patients with resected colon cancer, and its use as a quality measure will be maintained. The results of this study suggest that the implementation of a policy of reflexive re-dissection for colon cancer specimens with inadequate lymph node yields decreases the number of insufficient lymph node specimens and significantly improves compliance. 


\section{References}

1. Siegel RL, Miller KD, Jemal A. Cancer statistics, 2015. CA Cancer J Clin. 2015;65(1):5-29.

2. Compton CC, Fielding LP, Burgart LJ, et al. Prognostic factors in colorectal cancer. College of American Pathologists Consensus Statement 1999. Arch Pathol Lab Med. 2000;124(7):979-994.

3. Nelson H, Petrelli N, Carlin A, et al. Guidelines 2000 for colon and rectal cancer surgery. J Natl Cancer Inst 2001; 93(8):583-96.

4. American College of Surgeons: Cancer Program. Commission on Cancer Quality Measures. 2016.

5. Parsons HM, Begun JW, Kuntz KM, et al. Lymph node evaluation for colon cancer in an era of quality guidelines: who improves? J Oncol Pract. 2013; 4):e164-171.

6. Cianchi F, Palomba A, Boddi V, et al. Lymph node recovery from colorectal tumor specimens: recommendation for a minimum number of lymph nodes to be examined. World J Surg. 2002;26(3):384-389.

7. Bilimoria KY, Stewart AK, Edge SB, Ko CY. Lymph node examination rate, survival rate, and quality of care in colon cancer. JAMA. 2008;299(8):896; author reply 897-898.

8. Chen SL, Bilchik AJ. More extensive nodal dissection improves survival for stages I to III of colon cancer: a population-based study. Ann Surg. 2006; 244(4):602-610.

9. Le Voyer TE, Sigurdson ER, Hanlon AL, et al. Colon cancer survival is associated with increasing number of lymph nodes analyzed: a secondary survey of intergroup trial INT-0089. J Clin Oncol. 2003 21(15):2912-2919.

10. Swanson RS, Compton CC, Stewart AK, Bland KI. The prognosis of T3N0 colon cancer is dependent on the number of lymph nodes examined. Ann Surg Oncol. 2003;10(1):65-71.

11. Baxter NN, Virnig DJ, Rothenberger DA, et al. Lymph node evaluation in colorectal cancer patients: a population-based study. J Natl Cancer Inst. 2005;97(3):219-225.

12. Bilimoria KY, Bentrem DJ, Stewart AK, et al. Lymph node evaluation as a colon cancer quality measure: a national hospital report card. J Natl Cancer Inst . 2008;100(18):1310-1317.

13. Nathan H, Shore AD, Anders RA, et al. Variation in lymph node assessment after colon cancer resection: patient, surgeon, pathologist, or hospital? J Gastrointest Surg. 2011;15(3):471-479.

14. Markl B. Stage migration vs immunology: The lymph node count story in colon cancer. World J Gastroenterol. 2015;21(43):12218-12233.

15. Bui L, Rempel E, Reeson D, Simunovic M. Lymph node counts, rates of positive lymph nodes, and patient survival for colon cancer surgery in Ontario, Canada: a population-based study. J Surg Oncol. 2006;93(6):439-445.

16. Soreide K, Nedrebo BS, Soreide JA, et al. Lymph node harvest in colon cancer: influence of microsatellite instability and proximal tumor location. World J Surg. 2009;33(12):2695-2703.

17. Stocchi L, Fazio VW, Lavery I, Hammel J. Individual surgeon, pathologist, and other factors affecting lymph node harvest in stage II colon carcinoma. is a minimum of 12 examined lymph nodes sufficient? Ann Surg Oncol. 2011;18(2):405-412.

18. Benson AB, 3rd, Schrag D, Somerfield MR, et al. American Society of Clinical Oncology recommendations on adjuvant chemotherapy for stage II colon cancer. J Clin Oncol. 2004:22(16):3408-3419.

19. van Erning FN, Crolla RM, Rutten HJ, et al. No change in lymph node positivity rate despite increased lymph node yield and improved survival in colon cancer. Eur J Cancer. 2014;50(18):3221-3229.

20. Parsons HM, Tuttle TM, Kuntz KM, et al. Association between lymph node evaluation for colon cancer and node positivity over the past 20 years. JAMA. 2011;306(10):1089-1097.

21. Baxter NN, Ricciardi R, Simunovic M, et al. An evaluation of the relationship between lymph node number and staging in $\mathrm{p} T 3$ colon cancer using population-based data. Dis Colon Rectum. 2010; 53(1):65-70.

22. Wong SL, Ji H, Hollenbeck BK, et al. Hospital lymph node examination rates and survival after resection for colon cancer. JAMA. 2007;298(18):2149-2154. 